Document downloaded from:

http://hdl.handle.net/10251/146296

This paper must be cited as:

Torregrosa, AJ.; Broatch, A.; Pastor, JV.; Garcia Tiscar, J.; Sharma, RK.; Cheung, R. (12-2). Measuring turbocharger compressor inlet backflow through particle image velocimetry. Experimental Thermal and Fluid Science. 99:420-432. https://doi.org/10.1016/j.expthermflusci.2018.08.015

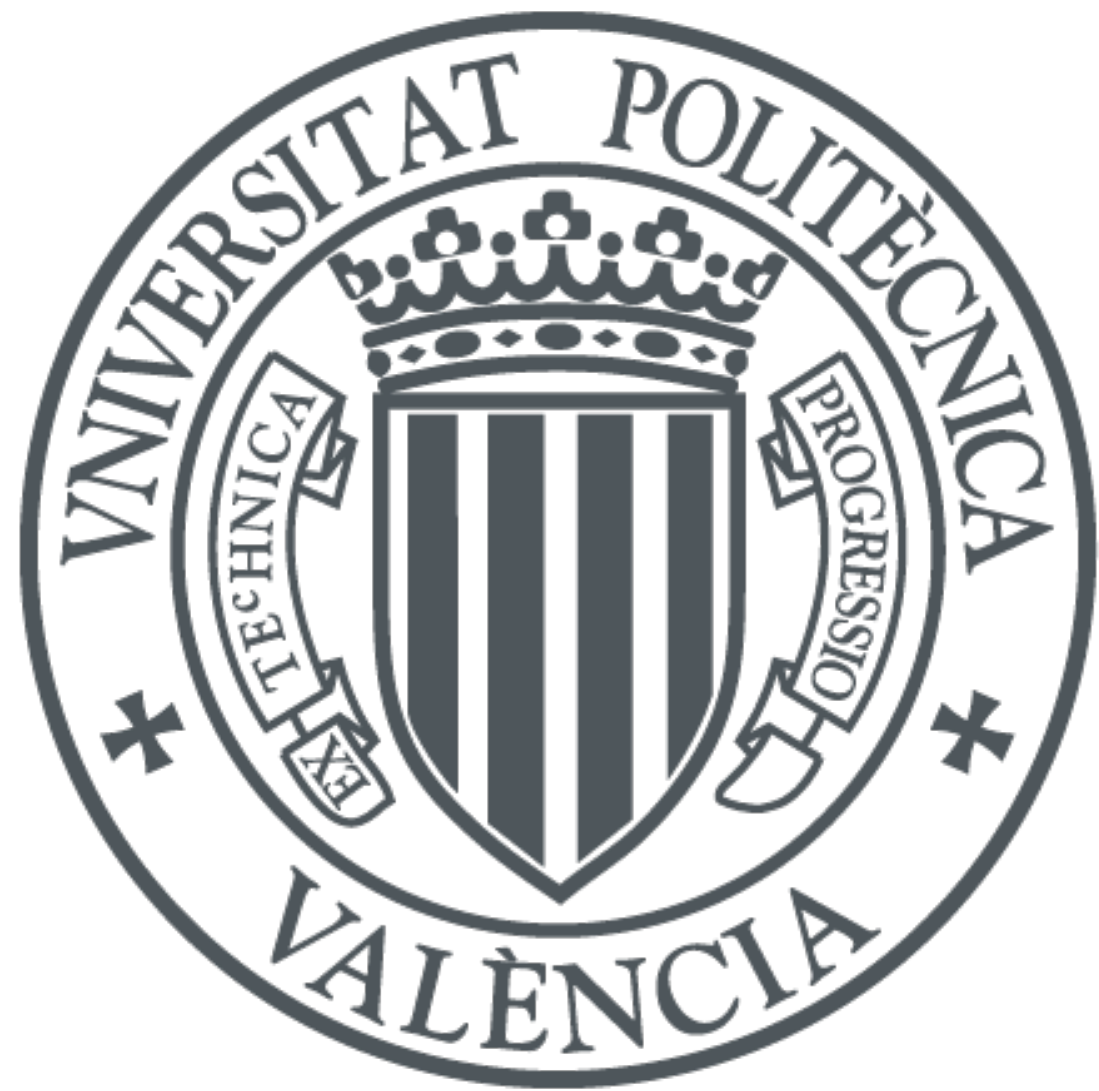

The final publication is available at

https://doi.org/10.1016/j.expthermflusci.2018.08.015

Copyright Elsevier

Additional Information 


\title{
Measuring turbocharger compressor inlet backflow through particle image velocimetry
}

\author{
A. J. Torregrosa ${ }^{a}$, A. Broatch ${ }^{\mathrm{a}}$, J. V. Pastor ${ }^{\mathrm{a}}$, J. García-Tíscar ${ }^{\mathrm{a}, *}$, R. K. Sharma ${ }^{\mathrm{b}}$, R. Cheung \\ ${ }^{a}$ CMT - Motores Térmicos, Universitat Politècnica de València, Camino de Vera, 46022 Valencia, Spain \\ ${ }^{b}$ Jaguar Land Rover Limited, Abbey Road, Whitley, Coventry CV3 4LF, United Kingdom
}

\begin{abstract}
An experimental measurement campaign is presented where particle image velocimetry (PIV) was used in an effort to characterize the velocity field in a turbocharger compressor when unstable operating conditions lead to flow reversing from the diffuser into the inlet pipe. Previous studies have successfully used this and similar techniques, but the most relevant results have been obtained in an open compressor where the backflow can diffuse into the ambient. In this work a glass pipe long enough to confine the full extent of the backflow has been used. Advantage was taken from the fact that this backflow is at higher temperature due to the compression process, enabling a preliminary work where a thermocouple array was used to estimate its maximum length across the compressor map. Using these results as a reference both axial and transversal velocity fields were measured. Issues associated with each one are described, along with relevant results that show how the technique correctly identifies the reversed flow, a conclusion that is supported by the comparison of the velocity average and standard deviation profiles with those of measured temperature.
\end{abstract}

Keywords: Turbomachinery, Flow visualization, NVH, Automotive, Surge, Stall

\section{Introduction}

Given the sustained push for the automotive industry to achieve greater efficiency and further emission reductions, thermal engines of passenger vehicles continue to undergo a transformation towards reduced engine displacement [1].

While these engines bring many advantages, they are not without issues. In order to meet power targets equivalent to those of their larger predecessors, the supercharging strategy of the downsized engine has become a cornerstone of its design and development [2]. On pair with engines, automotive turbocharger compressors have become smaller and, more importantly, are expected to provide higher levels of boosting pressures even at reduced air flow conditions [3].

As air mass flow is reduced at higher pressure ratios the compressor flow becomes unstable, ceasing to be evenly distributed. The impeller blades start stalling as flow detaches from their outer edges. This unstable operating regime impacts the efficiency of the compressor [4] and if mass flow rate is reduced too much or if changes in the operating point occur, as it is often the case with the sudden acceleration or deceleration of passenger vehicles, the compressor can go into deep surge.

A known issue since the early days of large steam-powered turbomachinery [5], deep surge occurs when the flow completely detaches from the impeller blades and, free of opposition, the compressed flow reverses direction and surges upstream the impeller and into the inlet duct until the momentum of the incoming air forces air back into the compressor, starting a new surging cycle.

\footnotetext{
*Corresponding author. Email address: jorgarti@mot.upv.es
}

This hazardous phenomenon does not, however, appear spontaneously. It was promptly observed [6] that surge only happens after the slope of characteristic curve (this is, the curve drawn by the pressure ratio as air mass changes at a given constant shaft speed) reaches a maximum and changes direction, the pressure ratio decreasing instead of increasing as flow is further reduced.

Sometimes called mild surge conditions [7], this change in slope is produced by blades partially stalling and hot compressed flow reversing along the periphery of the impeller and then reintegrating into the core flow as the central part of the impeller still keeps operating.

Unstable operation conditions where these complex backflows are present in the inlet zone have also been linked to an increase in the unpleasant noise emitted by the compressor $[8,9]$, a concerning issue for automotive manufacturers as public demand for quieter vehicles rises.

The accurate characterization and modelling of these unstable flow conditions is thus necessary to successfully tackle these issues and design corrective measures. Proposals include different geometries in the final part of the inlet such as elbows [10], tapered ducts [11] or volumes, boosting the flow swirl with the use of vanes [12] pre-rotation devices [13], or different ported shrouds [14] to guide and contain the backflow.

In parallel to these experimental strategies numerical models have been developed to simulate the operation of the compressor at these unstable conditions and the flow detachment, reversal and reintegration process $[15,16]$. However, it is necessary for both CFD simulations and experimental modifications to be validated regarding how their setup affects 


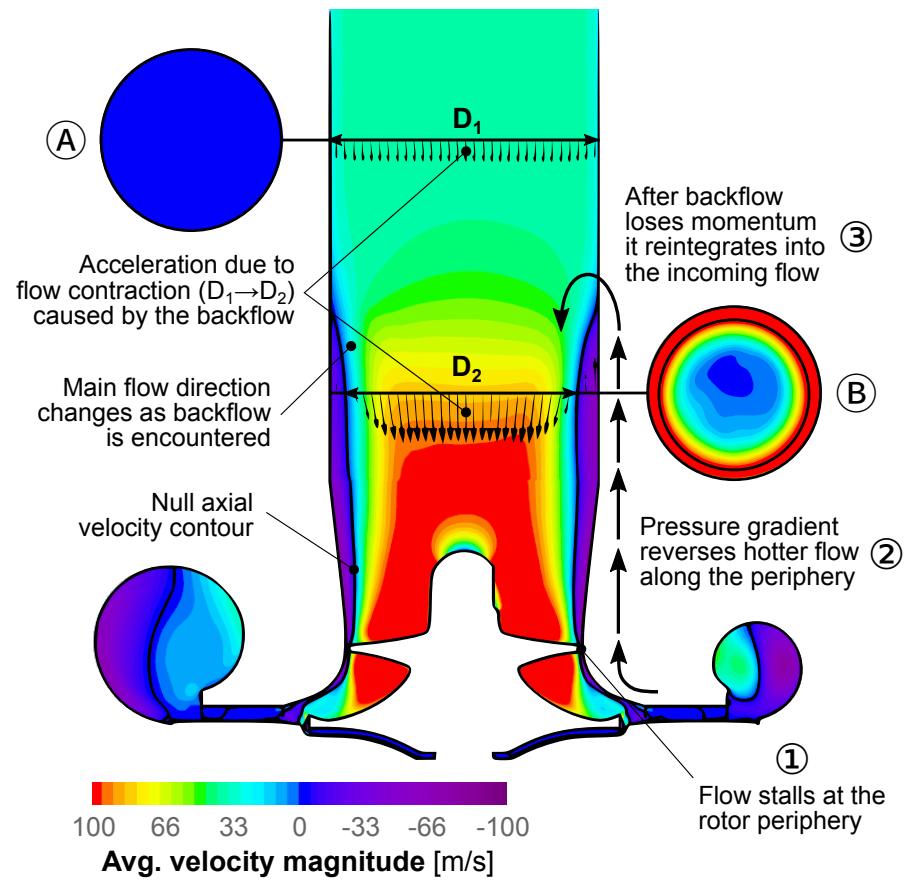

Figure 1: Numerical simulation of the velocity field at partially stalled conditions, describing the structure and fluid-dynamic mechanism of the backflow, and including transversal velocity slices: outside (A) and inside (B) the backflow.

the onset and the evolution of the backflow [17]. Gathering experimental data to support such validation is thus one of the primary objectives of the present work.

As an introductory example, in Fig. 1 results from a simulation presented in a previous work by the research group [16] are shown in order to illustrate the fluid-dynamic mechanism of compressor backflow. When operating conditions approach surge, flow stalls in the periphery of the rotor. Pressure gradient between the stalled and the compressed flow pushes the high temperature, compressed air upstream.

However, high shear against the incoming flow causes the backflow to rapidly lose momentum, and thus to reintegrate with the main flow after a certain length. As seen in the figure, reversed flow forces the main flow to change direction as it encounters the limit of the backflow, causing a contraction of the incoming flow and thus an increase in speed.

This unstable flow dynamic presents a challenge in numerical simulation, especially if industry-standard Reynoldsaveraged Navier-Stokes approaches are used. Thus, experimental validation of the backflow morphology (radii, extent, velocity) is a crucial step for the successful calibration and validation of CFD simulations.

Some indirect measurements have been made taking advantage of the temperature rise that the inlet experiences because of the high temperature of the compressed flow $[18,19]$. Low frequency contents and standard deviation of the pressure signals have also been used to track these unstable phenomena [20].

However, these methods do not resolve the spatial distri- bution of the backflow that is necessary to properly assess the effect of the different geometric modifications. In order to overcome this limitation, tests have been made to directly measure the flow velocity fields through different velocimetry techniques such as Laser Doppler Anemometry (LDA) [21], hot-wire anemometry (HWA) [22, 23] and Particle Imaging Velocimetry (PIV) [21, 24, 25].

The latter technique is desirable in this case as it allows the measurement of whole sections of the field instead of a single point such as in the HWA and LDA techniques. Regarding the use of PIV in turbomachinery, many of the studies available in the literature have been performed on large centrifugal pumps [21, 26, 27], axial fans [22, 28] and axial compressors $[24,25,29,30]$, usually with the aim to study the evolution of the flow through the passages and the interaction with the diffuser vanes.

In the case of the works presented by Liu et al. [25] and Wernet $[24,29]$ an effort was also made to analyze the distribution of flow in planes perpendicular to the channel direction so that the transversal velocity field could be observed.

Even when proper centrifugal compressors were studied, attention was still put in the diffuser passages, such as in the works of Voges et al. [31], Ohuchida et al. [32] and Cukurel et al. [33]. In the three cases a small transparent window is placed in the diffuser casing to record the passage flow and interaction with the impeller wake.

The most relevant studies that instead of the diffuser considered the compressor inlet and the aforementioned backflow issue are those published by Guillou, Gancedo et al. [34-37] who used a test rig where a turbocharger compressor was fitted with a bell mouth open inlet so that images of the flow could be taken directly without any barrier as the seeded ambient air was ingested. Hellström et al. [38] also used this setup to perform a comparison with numerical CFD simulations.

During these experimental investigations measurements of the transversal velocity fields were conducted by illuminating the flow outside of the inlet with a laser sheet perpendicular to the compressor axis and close to the bell mouth. An interesting development of this approach was made in [37] where a short polycarbonate ported shroud was fitted between the bell mouth and the compressor. The laser sheet was then made to pass at different planes through this transparent shroud.

On the other hand, axial velocity fields were measured in $[34,36]$ by aligning the laser sheet with the compressor axis, and taking the snapshots not perpendicular to it but at an angle, as the bell mouth was not transparent. By doing this with two cameras an stereoscopic algorithm could be used to reconstruct the true axial velocity field as if the camera were indeed perpendicular to the illuminated plane.

However, it must be noted that in real world automotive applications the compressor inlet is not open in this fashion but attached to a long duct that houses the filter and flow meter. These ducts confine the reversed flow and force its reintegration into the main flow. It has also been proved that the inlet geometry close to the compressor holds special relevance as it can influence the onset and frequency of the 
deep surge instabilities [39].

In this paper, an experimental effort is performed to measure the reversed inlet flow field when it is confined in a straight duct as seen in Fig. 2, that matches the inducer diameter and extends further enough from the compressor to allow the backflow to fully develop up to the extension that would be reached in realistic working conditions.

Thermal data measured by a thermocouple array will be be used to pinpoint the location of the backflow at different operating conditions, in order to better decide the setup of the PIV measurements. Both the experimental procedure for temperature and velocity measurements will be presented, together with a discussion of the obtained results that will show how the two kind of measurements relate and what conclusions can be drawn from this validation procedure.

\section{Experimental procedure}

\subsection{Turbocharger}

The turbocharger selected for the experimental campaign was a mid-sized automotive turbocharger. It was installed in the anechoic chamber of CMT-Motores Térmicos laboratories and powered from a nearby flow bench which is described in detail in [40]. Operating conditions of the turbocharger were regulated with the pressure of the air fed to the turbine and the operation of a back-pressure electro-pneumatic valve at the compressor outlet duct.

The pressure of the feeding line was adjusted using a screw compressor, powered by an asynchronous electric machine. Once the test turbocharger was working at the desired shaft speed, the back-pressure valve was used to restrict the airflow being ingested by the compressor.

Conditions were monitored through the in-house measurement and control system, which included outlet in-duct

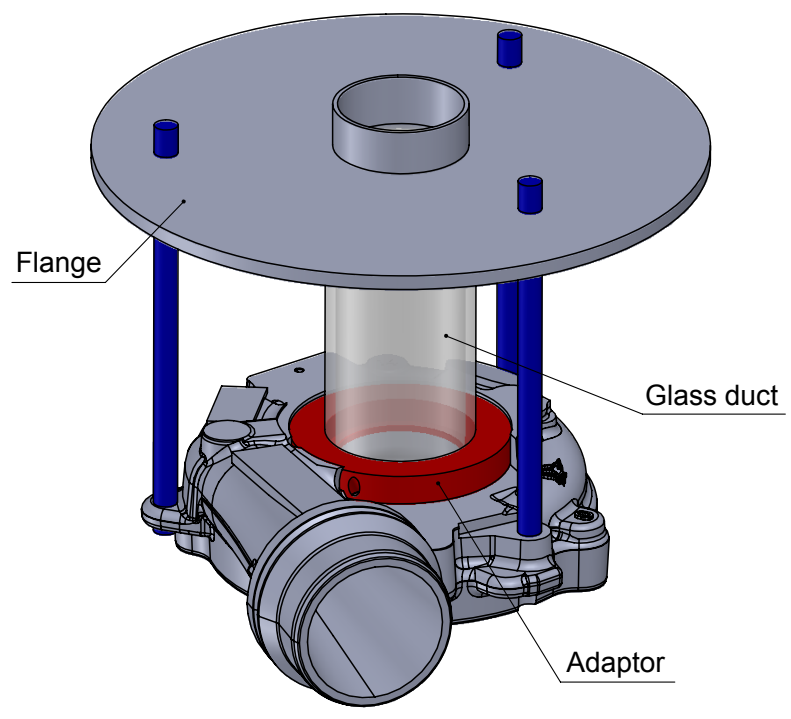

Figure 2: $C A D$ view of the straight transparent duct for PIV measurements with its mounting assembly. pressure and temperature and a Hall effect compressor shaft velocity sensor that detects each individual blade passing by their correspondent magnetic field disturbance.

\subsection{Laser PIV measurements}

Besides isolated measurements of pressure and temperature, the characterization of the whole flow field is also crucial. Not only for understanding how the backflow interacts with the downstream flow, but also, as discussed earlier, to validate CFD predictions of macroscopic flow structures such as those shown in Fig. 1.

In this study a standard 2D PIV system by TSI has been used for the measurement of the instantaneous 2D velocity fields. It consists of a double-head $15 \mathrm{~Hz} \mathrm{Nd:} \mathrm{YAG} \mathrm{laser}$ with $135 \mathrm{~mJ} /$ pulse @ $532 \mathrm{~nm}$, a set of mirrors and lenses to drive the laser into the measurement plane and change the laser output $5 \mathrm{~mm}$ circular beam into a laser sheet with around $1 \mathrm{~mm}$ thickness at the measurement section, a 4Mpixel, 12-bit straddle CCD camera (PowerView Plus 4MP) and a synchronization device. The air flow was seeded with $1 \mu \mathrm{m}$ mineral oil droplets from an atomiser using the Laskin nozzle principle (TSI 9306A Six-jet atomizer).

Images were processed with selected algorithms and validation criteria of the TSI Insight 3G software options. Postprocessing, graphical output and analysis tools were complemented with new processing routines developed in MATLAB for the specific purposes of this work.

The magnification of the images taken was around 30 $\mathrm{pixel} / \mathrm{mm}$ (in fact, between 28 and $32 \mathrm{pixel} / \mathrm{mm}$ depending on the arrangement) and the size of the interrogation area for calculation of the cross-correlations was set to $64 \times 64$ pixels, since the seeding was set at the minimum allowable in order to prevent oil accumulation, which forced excessive interpolation in some areas if smaller windows were used.

The authors chose to avoid relying excessively on interpolation since the objective of this work was not the minute analysis of the backflow but rather the macroscopic characterization of its onset, upstream extent and averaged velocities. These parameters are useful to validate and calibrate both numerical simulations and proxy experimental measurements of the backflow, such as those performed by means of temperature analysis discussed in this work.

The issue of oil soiling however is one of the major problems when dealing with confined compressor backflows, and will be discussed in detail in the following subsection. In any case, the selected window leads to the measurement of velocity fields with vectors separated a distance of 32 pixels, i.e. $\sim 1 \mathrm{~mm}$. Time elapsed between the two laser shots was set to a value between 2 and $10 \mu$ s depending on the flow velocities at each test point in the experimental test matrix.

Besides the issue of oil fouling, the possible issue of lensing effects due to the curvature of the duct was assessed by means of a calibration grid introduced into the duct prior to the starting of the turbocharger. Inspection of the grid demonstrated no significant lensing effect due to the curved geometry of the duct. A postprocessing test was made correcting possible 
deformations of the image but no differences could be appreciated against an uncorrected image. Therefore, this step was not considered in the postprocessing chain, although the calibration grid was still captured and inspected before each measurement in order to accurately convert from pixel space to real dimension space.

\subsection{Seeding at partial stall conditions}

Even during measurements of the longitudinal plane at stable conditions (high mass flow) where the stalled backflow does not reach upstream the leading edge of the blades, some of the seeding oil accumulated in the inner wall of the glass pipe, obstructing the view and enhancing reflections that rendered the measurement impossible through the affected parts. However, it is at partial stall conditions where the presence of the combined backflow depicted in Fig. 1 represents the biggest challenge for PIV measurements.

Fig. 3 shows a raw longitudinal snapshot, detailing how the detrimental reflections reduced the available region of interest (ROI) in which the velocity can be resolved. These reflections were mainly caused by the laser sheet entering and exiting the walls of the glass duct, which are visible in the image and annotated accordingly.

The issue was aggravated when the backflow generated by the partially stalled compressor blades reached the glass pipe. It can be seen in the previous figure how between the
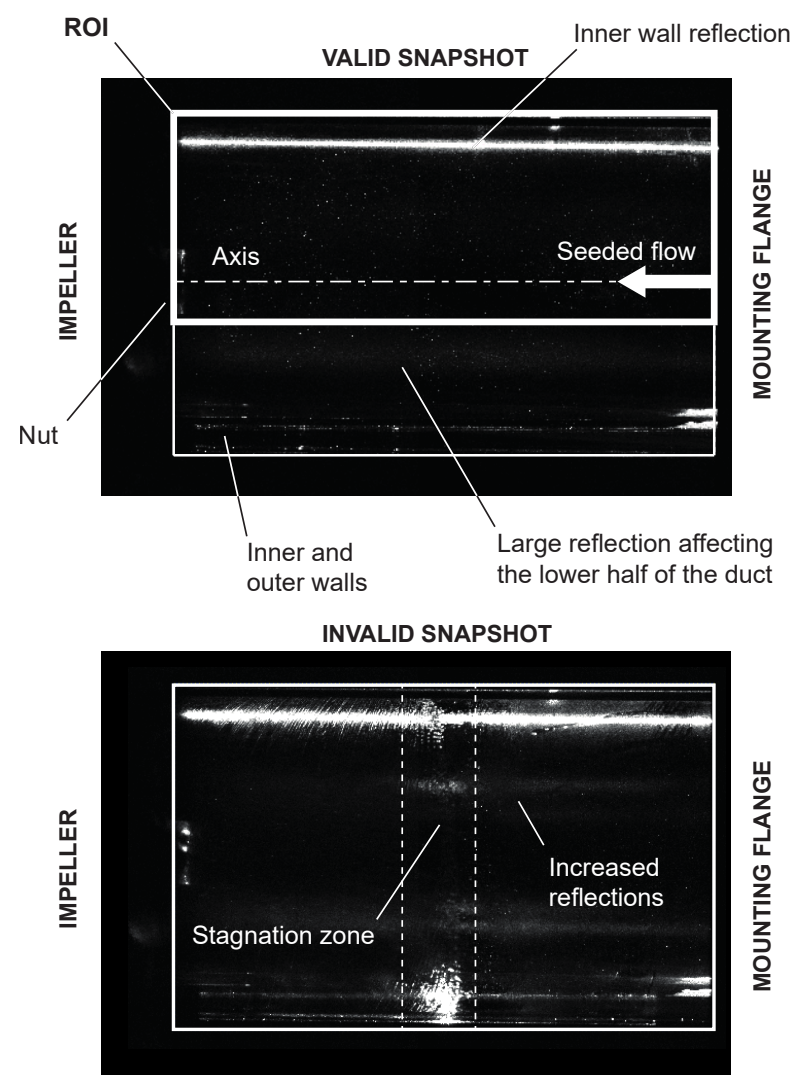

Figure 3: Raw images of the longitudinal plane showing a valid snapshot (top) and an invalid one (bottom) due to oil soiling.
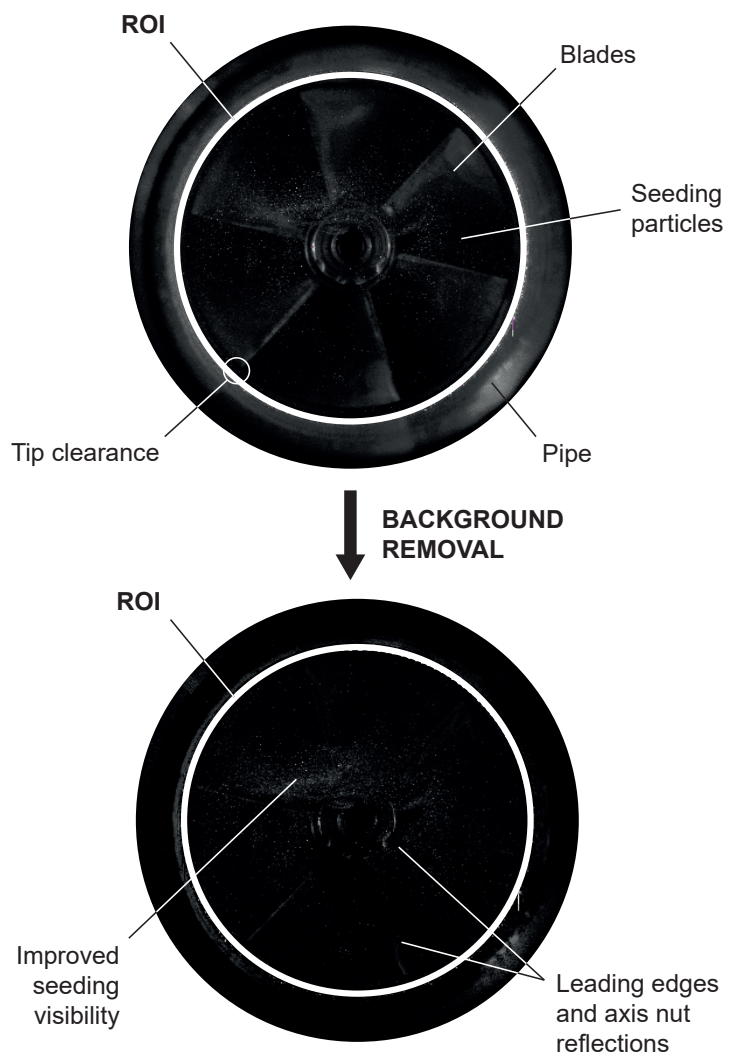

Figure 4: Raw snapshot of the transversal plane (top) and the same snapshot after the background removal postprocessing (bottom).

main flow and the backflow a null axial velocity contour is created. In the real compressor, this null axial velocity region is stopping the seeding particles along the periphery of the transparent duct, forming a clearly visible fluid "ring" that blocks the view, as seen in the bottom image of Fig 3.

Furthermore, previous numerical simulations predicted that this backflow keeps a high peripheral tangential speed as shown in Fig. 1 as well. Thus, the seeding particles are stopped by the null axial velocity and pushed against the walls by the tangential speed, impinging onto the inner wall and forming visible helicoidal tracks along the surface.

As a consequence, a test procedure was followed where the turbo was settled at the desired partially stalled operating conditions, and then seeding particles were introduced during at least $1 \mathrm{~s}$, and approximately 100 snapshot pairs of images were captured. This ensured approximately 10 oil ring-free pairs of snapshots, after which the oil accumulation due to the null axial velocity would affect measurements around the oil ring area. Once that the oil ring established, the turbocharger was stopped and the glass pipe cleaned for the next run.

Measurement of the transversal planes was done pointing the camera directly towards the compressor, thereby avoiding the need to record through the glass. This allowed for longer test runs because the soiling of the wall did not affect the 
measurement.

However, these measurements were not without issues. In this configuration, the rotor blades are illuminated by the scattered light as shown in the top image of Fig. 4, preventing the seeding particles from contrasting enough to be clearly correlated in some places, and generating bright spots that are misidentified as particles moving.

In order to mitigate this problem, a system was devised to feed the pulse train generated by the Hall effect RPM sensor into the laser and camera trigger controller, so that each pair of snapshots were taken at almost the same blade position.

This enabled a postprocessing technique where the average intensity of several non-seeded snapshots was subtracted from each seeded image, greatly reducing the brightness of the illuminated rotor and providing a better particle correlation, as shown in the bottom image of Fig. 4. In the case of the axial measurements, this was not deemed necessary since the back of the glass was painted matte and thus provided a good background.

While this resulted in overall enhanced quality of the reconstructed field, small reflections such as that of a small notch at the axis nut could not be removed. Also, in some occasions small glitches in the operation of the sensor resulted in pairs where the two laser pulses were included in the first frame, thereby rendering the pair useless. Fortunately, those cases were easy to identify and remove.

Since apart from the influence of the background the transverse velocity flow was much more chaotic than the longitudinal flow field, the quality of each individual snapshot pair correlation was worse than those of the axial case as the seeding particles were prone to "lump" together instead of homogeneously distribute, so only parts of the velocity field

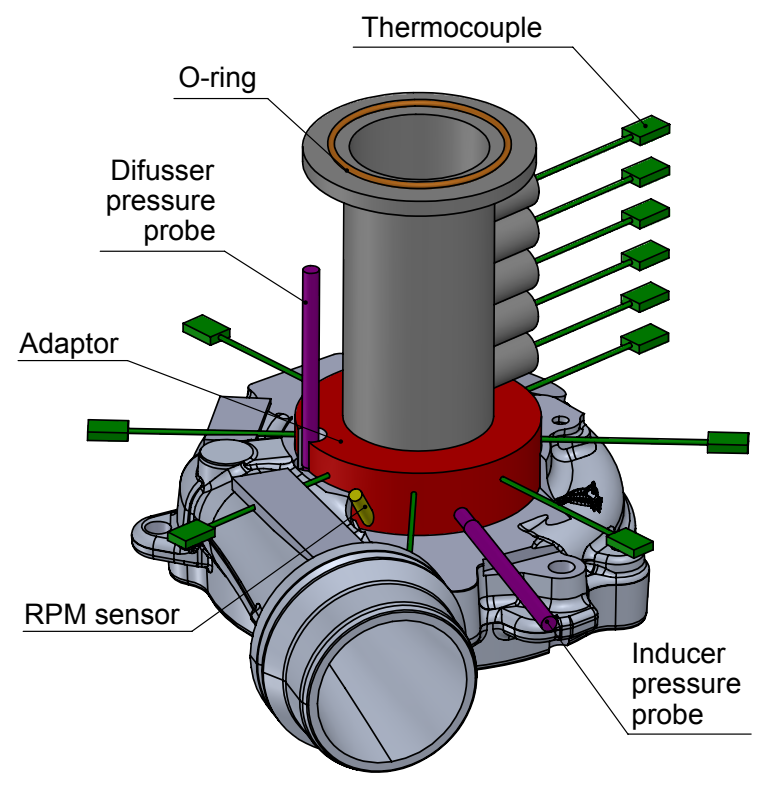

Figure 5: $C A D$ view of the local temperature and pressure measurement instrumentation, showing the distribution of thermocouple arrays and pressure probes. could be solved in each snapshot pair.

This effect can be seen in Fig. 4, and was one of the factors deciding the use of a $64 \times 64$ px interrogation window as smaller windows had made difficult to resolve valid vectors in the mostly empty areas left after the lumping of the seeding particles took place.

However, as the oil soiling of the wall was not as problematic as in the case of the axial velocity measurements, a larger number of valid snapshots could be selected (80-90 depending on the precise operating conditions) so that the averaged velocity field could still be solved with confidence.

\subsection{Temperature measurements}

In addition to the PIV measurements, a related measurement campaign focused on temperature distribution measurement had also been carried out for several different operating

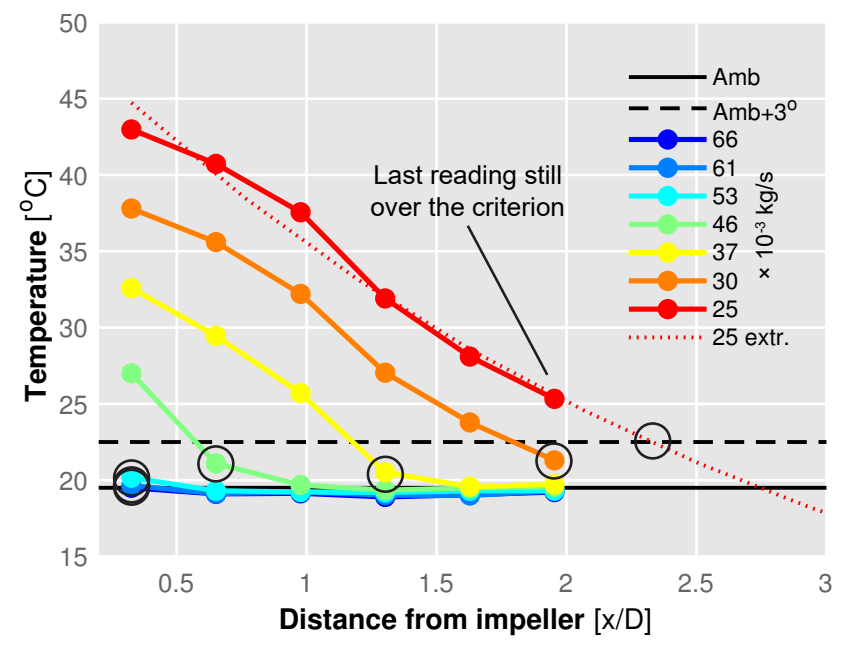

Figure 6: Temperature readings at the inlet linear array for a constant shaft speed $(80 \mathrm{krpm})$ and different mass flows. Circles indicate the estimated limit of the backflow for each mass flow using the proposed criterion.

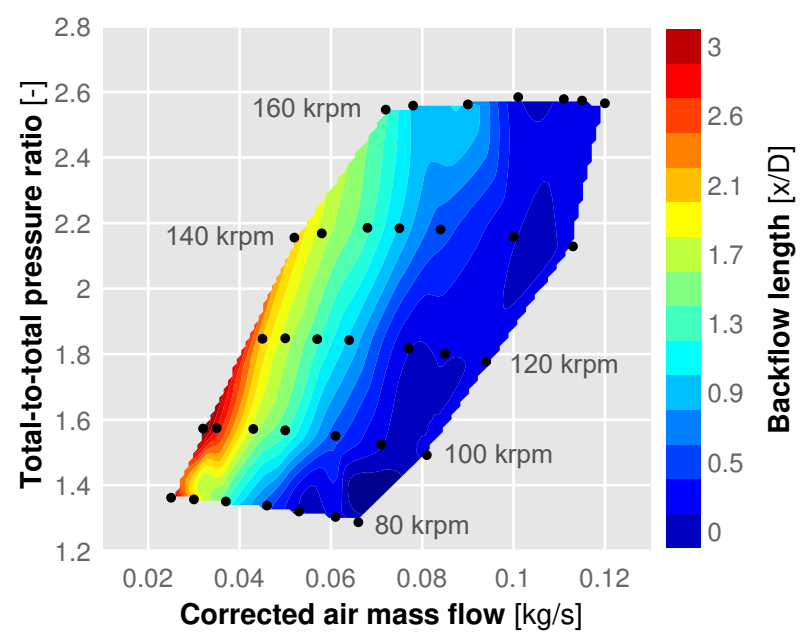

Figure 7: Interpolation of the backflow length estimated by the linear thermocouple array at different operating points of the compressor map. 
conditions in an effort to characterize the behaviour of the high temperature backflow over the most relevant part of the compressor map, this is, the mild surge region where the flow becomes partially stalled.

To this effect the turbocharger was fitted, among other sensors, with two thermocouple arrays: a circumferential one in order to detect temperature distribution skewness and a linear one along the inlet duct to detect the extension of this hot backflow. Both can be seen in Fig. 5 .

The first thermocouple of the linear array was located $1 / 3$ diameters (D) away from the leading edges of the compressor impeller. Subsequent thermocouples along the inlet use the same $1 / 3 D$ separation between them so the last one is located at $2 \mathrm{D}$ from the impeller. They were inserted into the flow up to half the radius of the duct (D/4) as in the work of Andersen et al. [18] in order to avoid wall heat transfer affects.

\section{Results and discussion}

\subsection{Temperature}

In order to estimate the length of the backflow a criterion was chosen where the location of the first thermocouple with a reading less than $3^{\circ} \mathrm{C}$ over the ambient temperature was regarded as the limit of the backflow extent for that operating point.

If the readings from all thermocouples were above this criterion, a log-linear extrapolation was used to estimate the rest of the temperature profile, regarding the point where the extrapolation intercepted the criterion as the backflow limit. Fig. 6 shows a sample of this criterion for all the measured points at a given shaft speed.

It can be seen in the figure that for the three higher mass flow points $(0.066,0.061$ and $0.053 \mathrm{~kg} / \mathrm{s}$ ) no temperature rise appeared, with the flow along the measured inlet section remaining at ambient temperature. As the flow was further restricted to $0.046 \mathrm{~kg} / \mathrm{s}$ a clear temperature rise was present, with the first thermocouple (counting from the compressor) being above the criterion. The rest of the array still measured flow at ambient temperature.

A similar situation happened for the next operating point at $0.037 \mathrm{~kg} / \mathrm{s}$, but for the next at $0.030 \mathrm{~kg} / \mathrm{s}$, which was already close to deep surge, the backflow appears to have extended to the end of the array, the last thermocouple barely meeting the criterion.

Lastly, at the lowest mass flow before deep surge $(0.025$ $\mathrm{kg} / \mathrm{s}$ ) it can be seen how every thermocouple read above the criterion, so a log-linear extrapolation (marked in a dotted line) was added to estimate how far beyond the array the backflow was extending.

This estimation was repeated for different shaft speeds, and at each one the air mass flow was varied from conditions of no backflow to as close as possible to the deep surge limit. Using these data points an interpolation was performed to cover the measured region of the compressor map. The result is shown in Fig. 7.

In order to construct the map, total pressures at both inlet and outlet were measured taking into account the air velocity and temperature, with the static pressure being captured by piezoresistive sensors mounted in the inlet and outlet duct at a suitable distance from the compressor in order to ensure that no flow perturbation affected the readings. The setup was equivalent to that used in related investigations by the group which focused on the relation between reversed flow and noise output $[41,42]$.

From the figure it can be seen that backflow was not present (or its length is less than $\mathrm{D} / 3$ ) for the higher mass flow conditions of each shaft speed isoline. Rather, backflow was detected when the slope of the characteristic line approached zero and increased as the flow decreased towards the surge limit.

It can also be seen how the length of the reversed flow

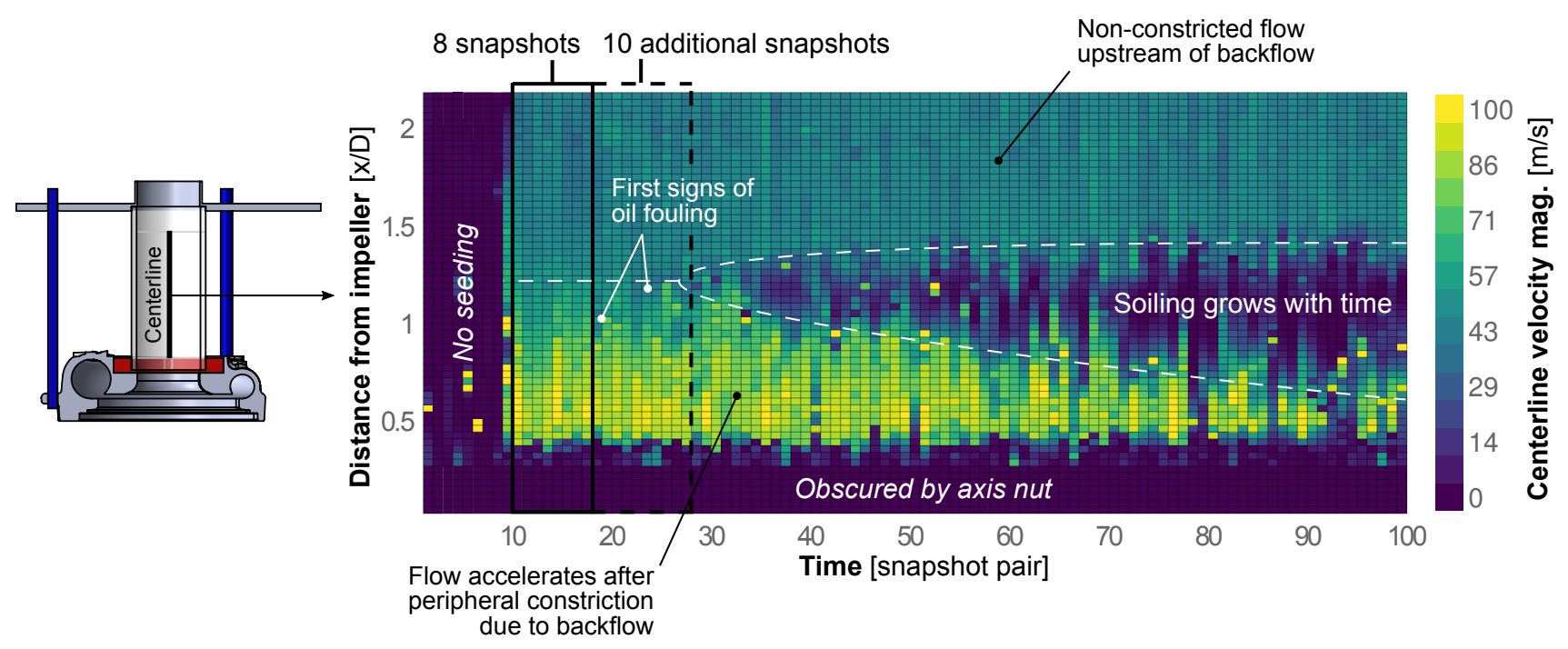

Figure 8: Temporal evolution of the longitudinal centerline velocity for the low air mass flow operating condition. 
was higher at the lowest shaft speeds than at the highest. This suggests that lower air mass flow and thus lower flow momentum allows the backflow to grow larger, even if the pressure gradient is considerably lower at these conditions. The map was thus used as a reference when performing the PIV measurements in order to estimate the upstream extent of the backflow that would be expected for each test.

\subsection{Velocity field}

\subsubsection{Longitudinal plane}

As described in the previous section, longitudinal plane measurements were the most difficult since the seeding oil particles blocked the line of sight of the camera. However with the right combination of seeding flow, oil dilution and quick operation of the system, valid snapshot pairs were obtained.

In order to capture the difference between stable and partially stalled conditions (the latter also called mild surge or marginal surge conditions) two back-pressure valve positions were tested, while maintaining the same shaft speed of 140 krpm.

PIV postprocessing was carried out for 100 snapshot pairs for each condition. However, while almost all of them where valid for the high mass flow condition, the aforementioned backflow oil trapping issue was critical in the low air mass flow case. In Fig. 8 the time-evolution of the centerline velocity is plotted to inspect this issue.

Eight valid flow snapshots $(10-17)$ are highlighted, followed by 10 more snapshots $(18-27)$ with small signs of fouling (sudden velocity deficit at some pixels) that could be included before the oil ring of Fig. 4 is fully established, causing a growing region of near zero velocity pixels (28-90).

Despite the growth of these soiled pixels upstream and (mostly) downstream of the backflow limit, two clear flow regimes could be distinguished before and after this limit. Upstream of the backflow and its subsequent soiled region, the flow was not constricted and showed a stable velocity. This regime appeared clearly constant for all 90 snapshots with seeding.

The figure also shows how after the flow reached the backflow limit, its velocity gradually and smoothly increased. This is of course more clear in the first $(10-27)$ snapshots, but even in the last snapshots $(>90)$ where the soiling is widespread, it is clearly seen that the flow consistently reached velocities of around $100 \mathrm{~m} / \mathrm{s}$.

Therefore, snapshots $10-27$ were selected for averaging the flow field at unstable, low mass flow conditions. On the other hand, for the high mass flow case, all 90 snapshots with seeding were valid, since no oil ring formed as the backflow was absent at stable conditions. The two resulting averaged
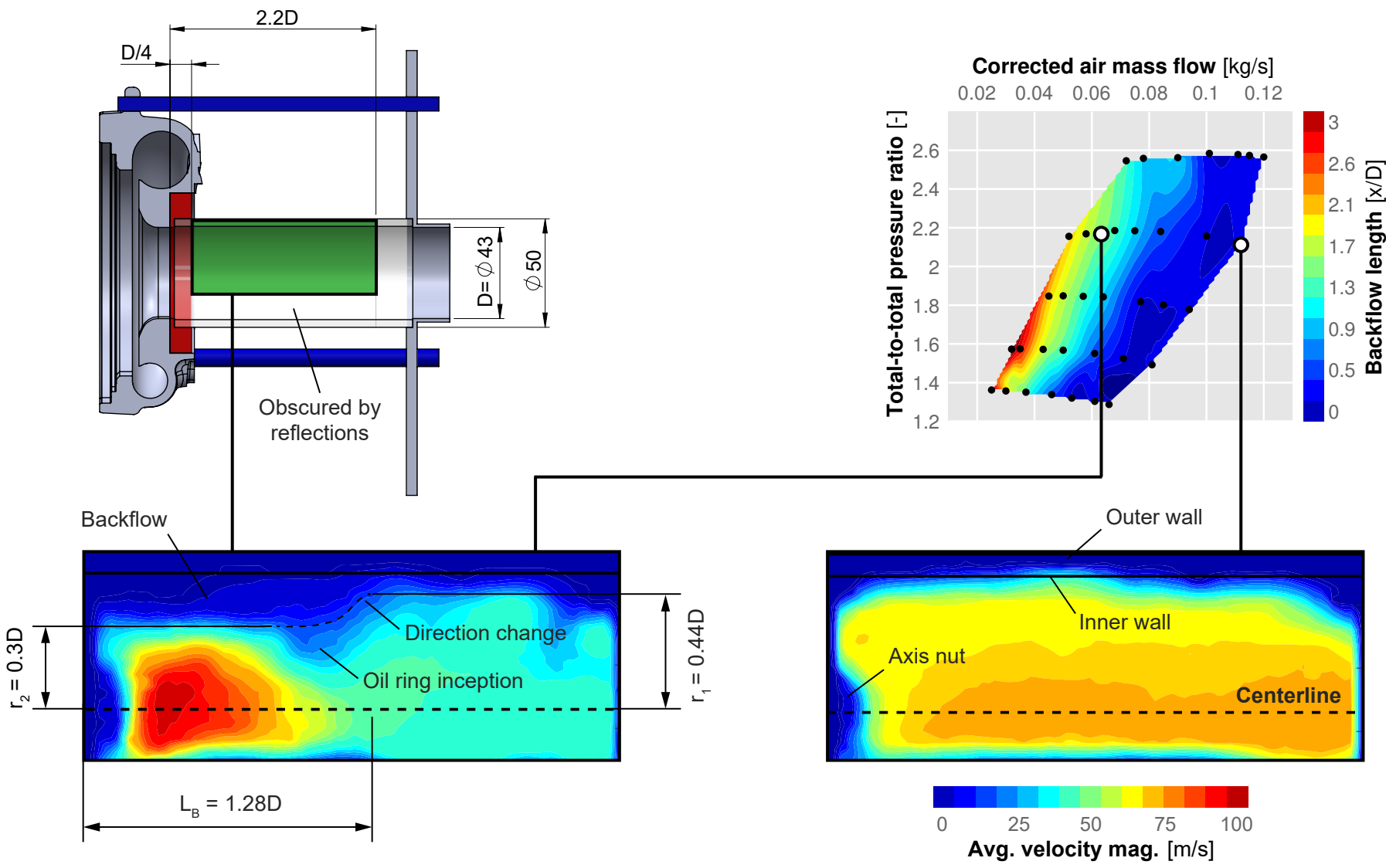

Figure 9: Results of the axial velocity field measurement (longitudinal plane) for the two operating points considered: low air mass flow (left) and high air mass flow (right), indicated along the estimated backflow length in the compressor map (top right). 

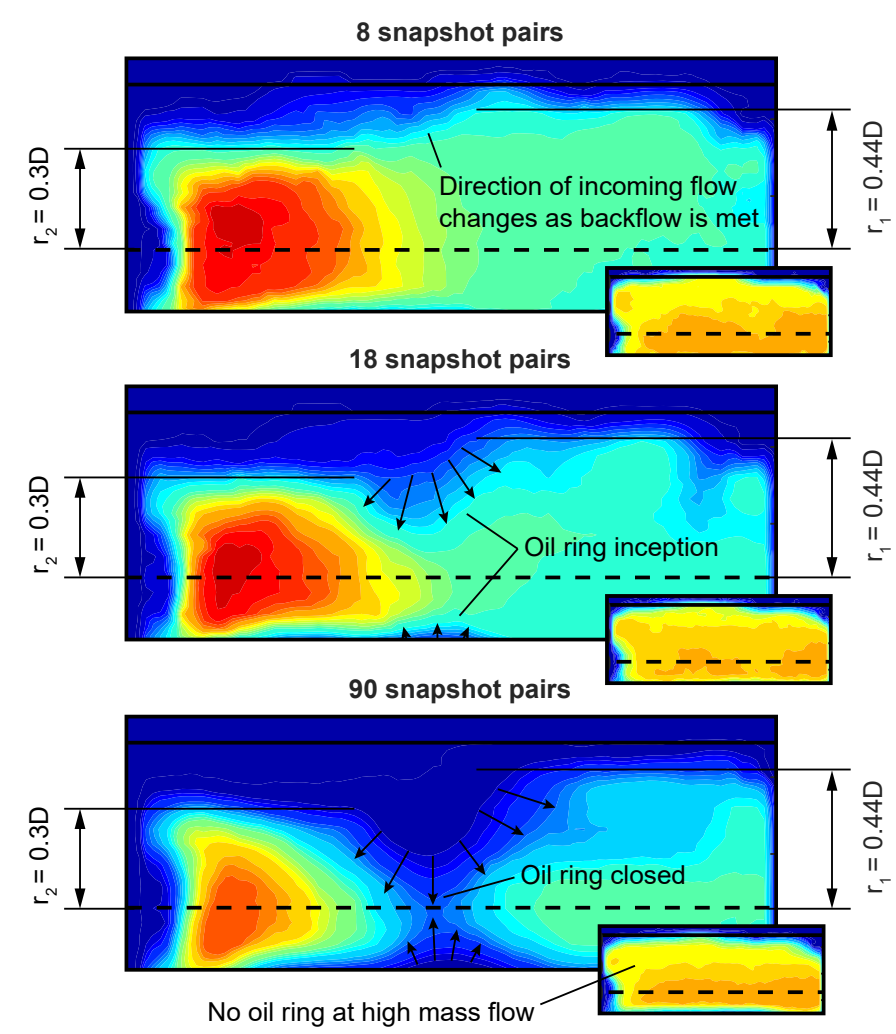

Figure 10: Temporal evolution of the longitudinal velocity field at low mass flow conditions, plotted by averaging an increasing number of snapshots. The same is done for the high mass flow point in the insets at the lower right corners.

velocity fields can be seen in Fig. 9. While the lower third of the frames were obscured by a reflection, at least $2 / 3$ of the axial plane could be resolved in sufficient quality.

In these tests there was no direct measurement of inlet air mass flow, so an average of the measured velocity field in the upstream area of the duct was taken to identify the precise operating point of the compressor.

At the higher mass flow, it can be seen in the figure that the velocity field was approximately homogeneous along the duct and only near the wall the velocity diminished. However, when flow was reduced, the spatial distribution of velocity changed. The expected compressor backflow was apparent as the low velocity region near the wall increased in thickness, reducing the useful diameter from $\sim 0.44 \mathrm{D}$ to $\sim 0.3 \mathrm{D}$.

This apparent reduction in velocity magnitude was probably caused by the high transverse velocity of the backflow, that makes the transit time of the particles through the laser sheet too small to be captured in consecutive snapshots and thus makes the correlation impossible so no velocity is detected, although as shown in Fig. 1 high reversed axial velocity should be present.

The reduction in diameter extended to approximately $1.28 \mathrm{D}$, this is, approximately half of the transparent glass length. This distance is consistent with the prediction made through temperature measurements as shown in Fig. 7, which is included for convenience.
It can also be seen in Fig. 9 how this reduction in the useful diameter appeared to be paired with an increase in the flow velocity, as predicted by the CFD simulation shown in Fig. 1 . A similar but lower increase can be seen in the work of Fike et al. [28] in a single channel of an axial flow fan. Likewise, a similar constriction and acceleration of the flow is correctly predicted by the compressor numerical model developed by Lang [43].

In order to ensure that the reduction of diameter is well captured by the PIV measurement, as it is one of the most relevant values for CFD calibration and validation, in Fig. 10 the temporal evolution of the longitudinal velocity field at the low mass flow condition is plotted by averaging, from top to bottom, snapshots $10-17,10-27$ and $10-100$.

In the 8 fouling-free snapshots $(10-17)$ the backflow is clearly defined and its morphology matches the numerical prediction shown in Fig. 1. However, when the next 10 snapshots are added, a slight growth of velocity deficit is seen extending from the limit of the backflow. The center of the pipe is still unaffected.

As the rest of the snapshots are added, the oil ring develops fully, extending from the initial inception point and obscuring the velocity readings (although the downstream velocity increase is still perceptible). In all three cases $r_{1}$ and $r_{2}$ are consistent, thereby demonstrating that even if the oil ring affects the quantification of the velocity, the morphology of the backflow contraction is still well captured.

On the other hand, no oil ring formed in the case of the high mass flow point, shown in a small inset for each number of averaged snapshots in Fig. 10. This is due to the absence of

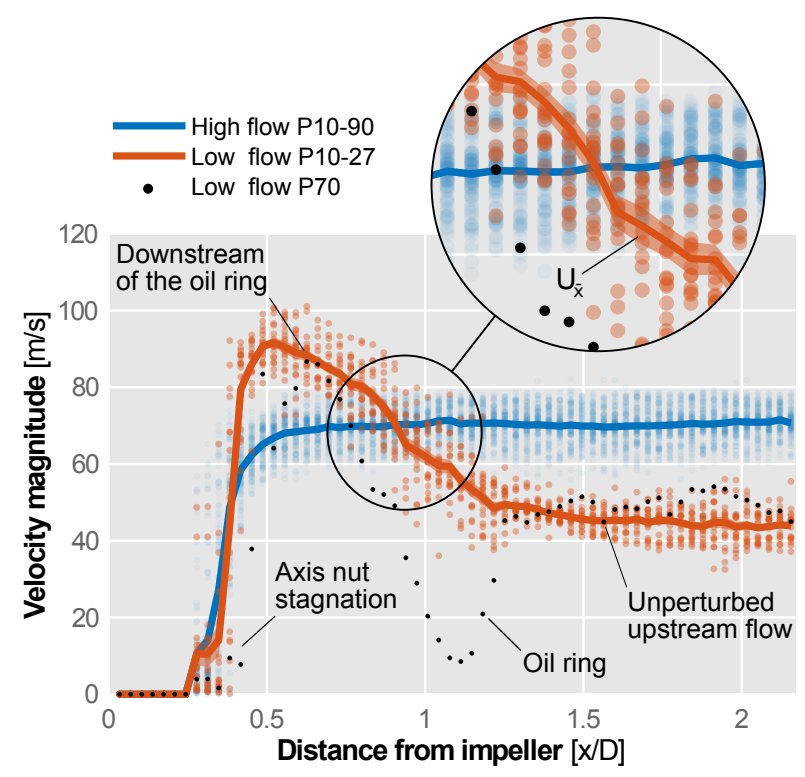

Figure 11: Velocity magnitude over the centerline of the longitudinal plane at high and low flow. Solid line indicates the mean and points correspond to individual snapshot pairs. Pair 8 is presented separately as it starts showing the effect of the oil fouling. Note that distances below $D / 4$ are obscured by the adaptor piece. 
null-velocity reversed flow zones in the periphery at these stable condition. Thus, the 90 seeded snapshot were considered for analysing operating condition.

To better quantify the increase in speed in the core flow, velocity data along the turbocharger axis line (marked with dashed line in Fig. 9) from each valid snapshot has been plotted in Fig. 11 for both operating conditions. An invalid snapshot for the unstable condition (snapshot pair 70) has also been plotted (black dots) to show how the oil film accumulating in the pipe wall due to the backflow affected the velocity measurement, especially at the oil ring position. Downstream the oil ring, its velocity started to recover, even reaching the average of the more clean $10-27$ snapshots.

While at the high flow operating point the flow exhibited an approximately constant velocity along the line, only decreasing adjacent to the axis nut, at the lower flow condition it can be seen how after a steady initial segment the velocity increased to a top average speed of $91.7 \mathrm{~m} / \mathrm{s}$. The average speed at the initial segment between 1.9 and 2.1D was found to be $43.7 \mathrm{~m} / \mathrm{s}$.

Uncertainty of the mean velocity $U_{\bar{u}}$ [44] has been calculated and added to the figure by taking into account the standard deviation $\sigma_{u}$ and the number of selected samples $N$ for each case as:

$$
U_{\bar{u}}=\frac{\sigma_{u}}{\sqrt{N}}
$$

Previously, Saphiro-Wilk tests were performed to check the normality of the experimental data, in addition to visual inspection of quantile-quantile (Q-Q) plots. Only the measurements of the transversal field outside of the backflow failed the normality test, as expected, due to the tangential velocity in the unperturbed incoming flow being practically null as predicted in Fig. 1.

As the parameter $U_{\bar{u}}$ is low in comparison with the mean, the centerline velocities shown in Fig. 11 appear to be reliable, more so as they appear consistent with the morphology of the backflow described by the aforementioned numerical simulations.

Thus, it is possible to draw a simple correlation between the area constriction and the increase of velocity. Using the mass conservation equation between the initial segment (denoted by the subscript 1 ) and the top velocity point (denoted by the subscript 2):

$$
\dot{m}_{1}=\dot{m}_{2}=\rho_{1} V_{1} \pi r_{1}^{2}=\rho_{2} V_{2} \pi r_{2}^{2}
$$

If a small enough increase of temperature between point 1 and point 2 is assumed so that $\rho_{1} \sim \rho_{2}$ the expression can be rearranged to obtain the velocity change:

$$
\frac{V_{1}}{V_{2}}=\frac{r_{2}^{2}}{r_{1}^{2}}
$$

If now the aforementioned approximate values for radii and velocities are substituted into the equation, we can obtain an estimation of the relative error $\varepsilon$ between the theoretical value and the experimental observation:

$$
\varepsilon(\%)=\left|\frac{V_{1} / V_{2}-r_{2}^{2} / r_{1}^{2}}{r_{2}^{2} / r_{1}^{2}}\right| \cdot 100 \approx 1.7 \%
$$

The small relative difference between the prediction and the observation supports the assumption that the measurement technique is in fact able to characterize the reduction in useful area associated with the backflow and its associated increase in velocity, even if the axial speed of backflow itself cannot be quantitatively measured.

\subsubsection{Transversal plane}

After the measurement of the axial velocity field at the longitudinal plane, the setup was modified to measure transverse planes. The camera was moved to point directly through to the compressor short inlet pipe, while the cylindrical lenses were rotated $90^{\circ}$ to create a vertical laser sheet.

During this test, the contrast between a high flow and a low flow operating condition was again measured as in the axial case, in order to ensure that the method allowed for a clear differentiation between the backflow coming from downstream the impeller and the unperturbed, non-rotating flow coming from upstream the compressor.

As mentioned earlier, in contrast with the longitudinal case, the background of the snapshots for these transverse measurements had to be necessarily the rotating, polished turbocharger impeller itself instead of the back of the glass pipe. This caused an increased amount of bright reflections that made tracking of the seeding particles more difficult.

To reduce the severity of this issue, snapshots were synchronized with the blades' position through the existing shaft speed Hall effect sensor. However, the data transfer rate of the particular camera used in this test limited the time between snapshots and thus the shaft speed at which the synchronization mechanism operated correctly. A speed of $100 \mathrm{krpm}$ was selected as a compromise between safe operation of the synchronization mechanism and adequate expected length of the backflow.

Using this setup a first test was carried out where the transversal plane illuminated by the laser sheet was located at approximately 1.2D from the compressor impeller leading edges, since at this distance it was expected to adequately capture the backflow as per the previous temperature measurements.

Approximately 100 velocity snapshots ( 90 with valid seeding) were captured for both the low flow and the high flow operating conditions. Since in this case the accumulation of oil along the walls of the duct was not such a relevant issue they were all averaged, after subtracting a reference background image made from the average of the first 10 snapshots pairs, captured before the seeding started as shown in Fig. 4. The results of this measurement are shown in Fig. 12.

In this figure, the two operating conditions are marked on a compressor map that estimates the length of the high temperature backflow. As expected by taking into account the numerical predictions, the tangential velocity at the high flow 


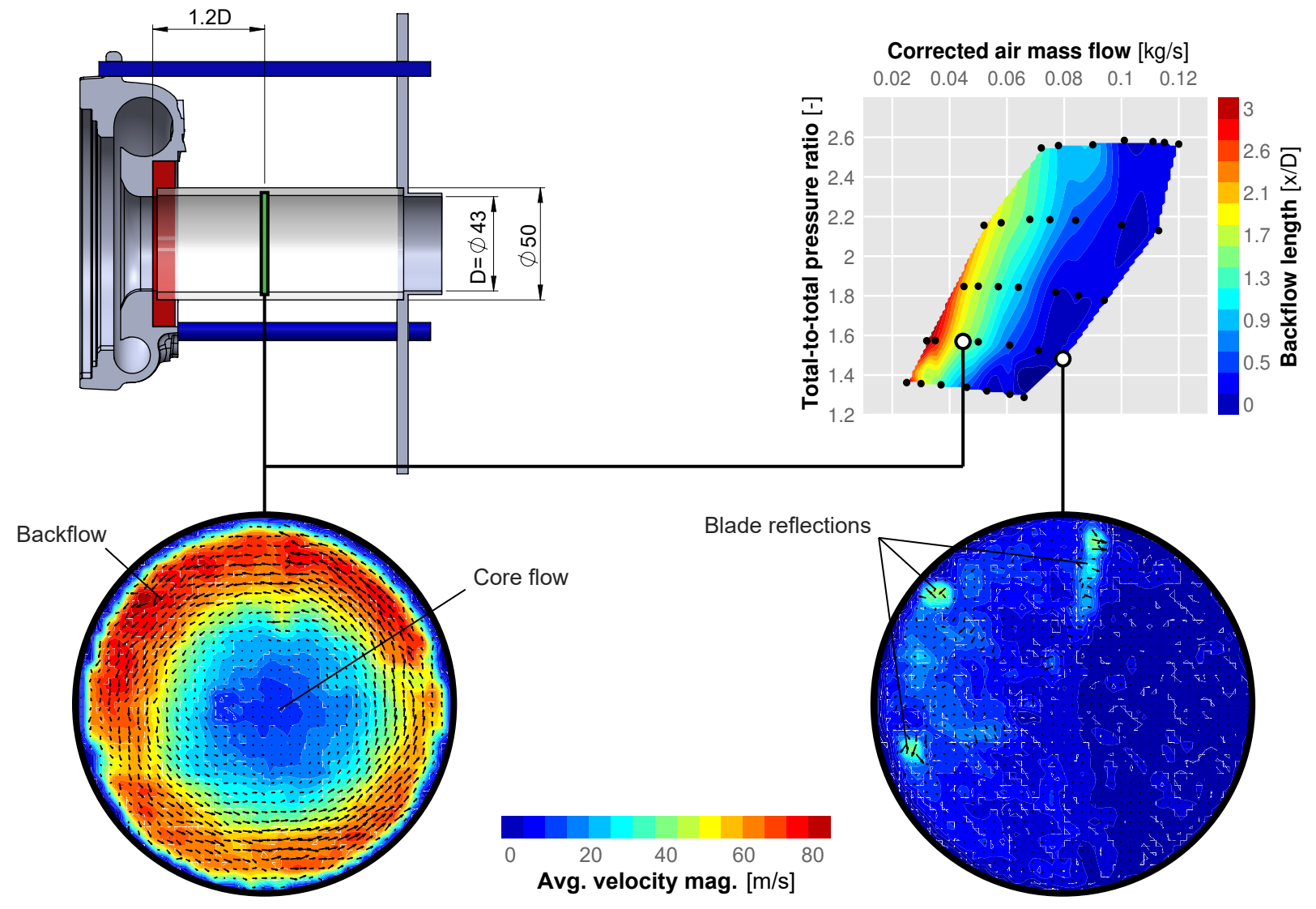

Figure 12: Results of the averaged transversal velocity field for two operating points: low air mass flow (left) and high air mass flow (right), indicated along the measured inlet backflow length in the compressor map.

case was very low, albeit some reflections from the impeller blades are showing up in the correlation. This was one of the only cases where the Saphiro-Wilk test of normality failed, since no meaningful data was being captured.

For the low mass flow rate case, which makes the compressor operate at a condition where a high backflow is expected, a clearly different flow field can be observed. In this case there was a highly rotational field near the walls that diminished in the direction of the core flow, coherent with the backflow coming back through the stalled tips of the impeller blades and through the tip clearance itself, as shown in Fig.1.

As expected, the PIV postprocessing method could not resolve the flow field up to the glass pipe wall, as there was a small zone where the correlation did not give valid results, since the rectangular grid did not contain enough seeding particles as they were slowed down by the boundary layer and obscured by the wall reflection.

After attesting that the experimental setup was able to capture the rotating backflow, a final test was made where the turbocharger was kept at an approximately constant low flow operating condition and the laser sheet was placed at different distances along the glass inlet pipe in order to measure how the backflow-induced rotational field evolved along the pipe.

The selected operating condition was again at $100 \mathrm{krpm}$ of shaft speed and a low flow point similar (but not equal) to that of Fig. 12. Three different snapshots sets were taken with the laser sheet at distances of $\mathrm{D} / 2,1 \mathrm{D}$ and 2D from the leading edges of the impeller blades. Expected length of the compressor backflow at these conditions was around 1.6D according to the temperature profile data. Results of this study can be seen in Fig. 13.

In this figure, two of the slices (left and center) were inside the backflow, as expected, while the third one (on the right) was clearly situated outside the backflow as the rotational velocity appeared almost null. The later was the only other case where the normality test failed, as the velocity magnitudes are too small to be meaningful.

It is also apparent that the slice closest to the impeller (D/2) exhibited a thicker zone of stronger rotational velocity, starting from the wall and diminishing towards the centre. A reflection coming from a dent in the axis nut (probably made for balance) and some slight reflections from the blades is also visible.

The second slice at 1D from the leading edges featured a similar velocity field, albeit a slight reduction in both the speed and the thickness of the backflow is appreciable. The effect of erroneous reflections from the blades is also stronger in the core flow section.

To quantitatively compare the velocity profiles of the three slices, velocity magnitude data was extracted from the hor- 


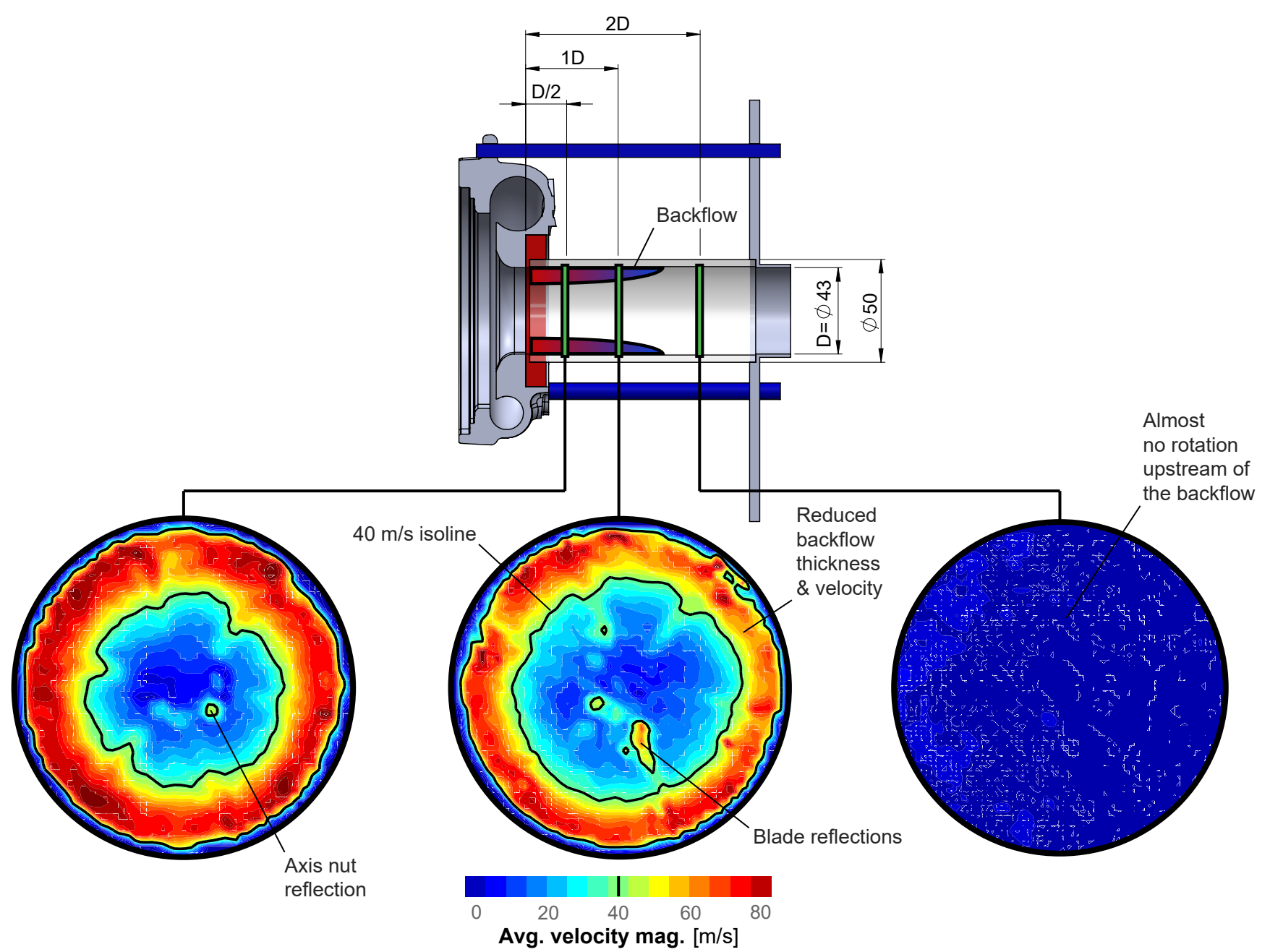

Figure 13: Results of the averaged transversal velocity magnitude for three slices at different distances from the compressor impeller leading edges. From left to right $D / 2,1 D$ and $2 D$ distances. Same compressor operating condition for all slices.

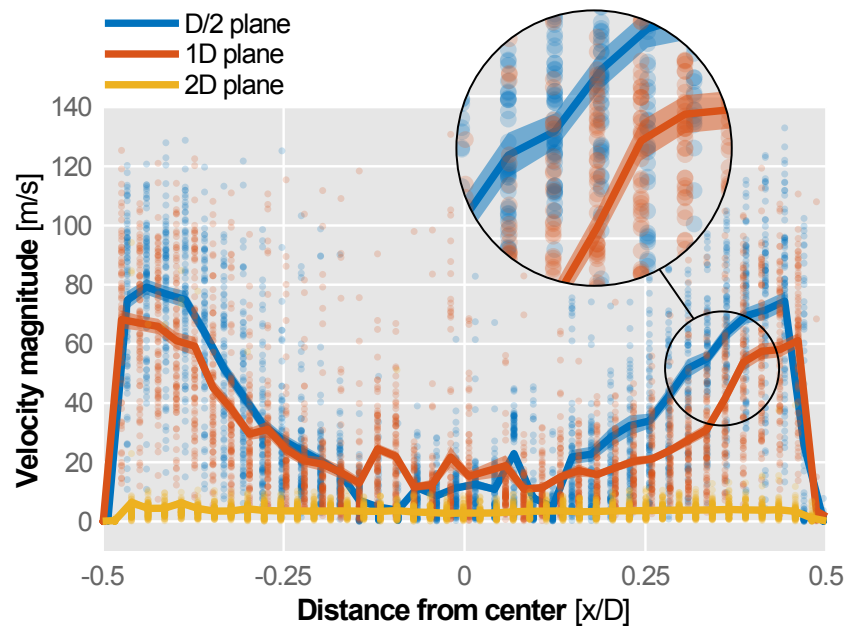

Figure 14: Velocity magnitude over the centerline of three transversal planes. Solid line indicates the mean and points correspond to individual snapshot pairs. izontal centerline of each slice. Fig. 14 shows the velocity data for each individual snapshot (points) and the averaged magnitude (solid line), including its uncertainty computed with Eq. 1.

Dispersion at each point of the line was much higher that in the previously discussed case of longitudinal plane measurements, but it could be confirmed that in average the 1D slice presented lower top speed and less thickness: any given speed (for instance, $50 \mathrm{~m} / \mathrm{s}$ ) was achieved closer to the wall than in the $\mathrm{D} / 2$ slice.

In the case of the $2 \mathrm{D}$ slice which as predicted was located outside of the backflow, velocity and dispersion were much lower, the profile being constant at an approximate magnitude of $4 \mathrm{~m} / \mathrm{s}$, probably due to pure momentum diffusion upstream but in any case not with a meaningful rotation.

Core flow rotation velocity appeared to be similar in the three measured slices, at least at radius of less than $0.2 \mathrm{D}$, although the reflections of the blades and the axis nut introduced some perturbations in the $\mathrm{D} / 2$ and $1 \mathrm{D}$ slices. On the other side, velocity could not be resolved at locations with radius greater than $0.56 \mathrm{D}$, leaving a gap of missing data of 

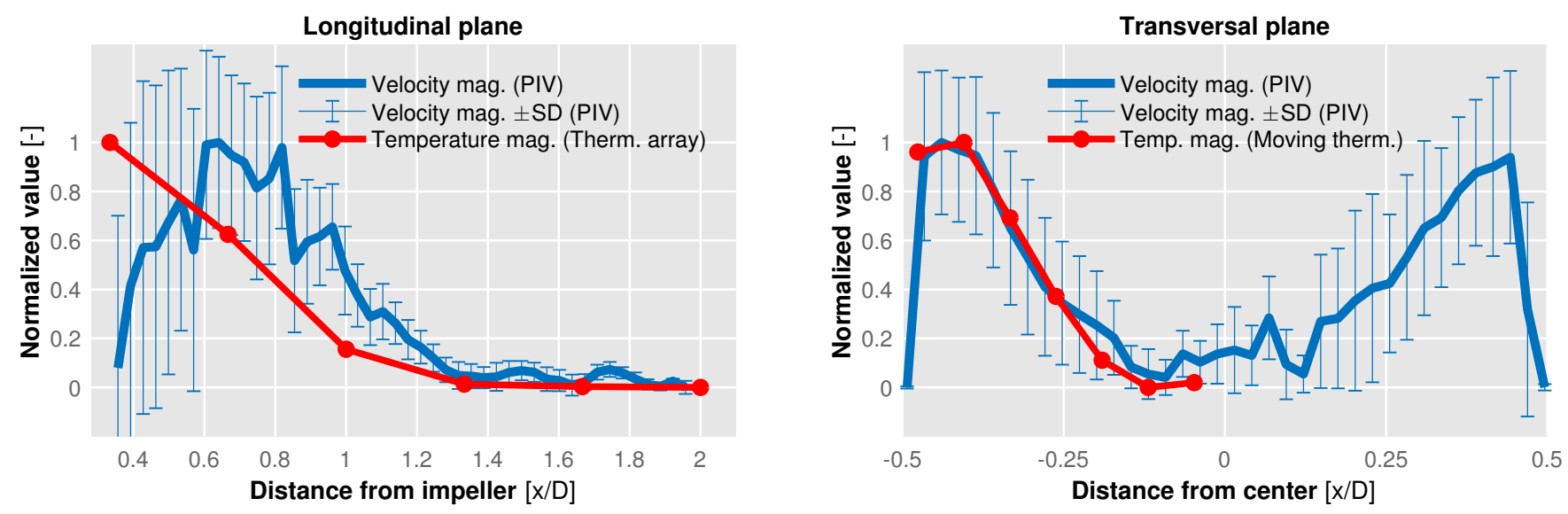

Figure 15: Normalized profile comparison between temperature measurements and PIV results on both the transversal plane (right hand plot) and the longitudinal plane (left hand plot).

about $0.03 \mathrm{D}(1.5 \mathrm{~mm})$ from the pipe wall.

\subsection{Comparison with temperature}

Besides using the thermocouples described in section 2.4 for estimating the backflow extent across the different operating conditions, a comparison was performed between the velocity profiles obtained through PIV and the averaged temperature profiles obtained through the arrays shown in Fig. 5.

First a velocity profile was extracted from the axial plane at a line separated $\mathrm{D} / 4$ from the wall, which was the distance at which the thermocouples were installed. Since at this distance, closer to the top of the field of view the oil ring, inception shown in Figs. 9 and 10 was present after 8 seeded snapshots, only snapshots $10-17$ are considered in this section in order to avoid the spurious velocity deficit caused by the oil ring.

After selecting these valid snapshots, velocity magnitude profile was normalized between 0 and 1 , and the same was done with the thermocouple array data that most closely matched the operating point at which the PIV test was performed. The results can be seen in the left plot of Fig. 15. In addition to the temperature profile and the averaged value of the velocity magnitude, error bars indicating the standard deviation values are included.

Contrasting with Fig. 11 where the centerline profile was shown to have a relatively small dispersion, at the thermocouple array location (half a radius from the wall) the profile suffered from a much larger deviation at locations where the velocity increased towards the compressor.

Compared with the normalized longitudinal temperature profile measured by the thermocouple array however, it can be seen that there is a good agreement in the maximum extent of the backflow influence on both velocity and temperature, with the turning point located around 1.3D from the impeller and velocity and temperature then increasing towards the impeller.

To compare the radial profile of the backflow in terms of velocity and temperature, a test was performed where the turbocharger was kept at a certain fixed operating condition while the thermocouple closer to the impeller was progressively inserted into the air stream, from close to the wall to the centerline. Again, data was normalized so that both variables ranged between 0 and 1 . The results can be seen in the right hand side of Fig. 15.

In this case the match between temperature and transversal velocity profiles is even better than in the axial case: near the wall there was a small section where temperature and velocity were maximum and then both diffused towards the center, reaching a stable value corresponding approximately to the core flow at $\pm 1 / 8 \mathrm{D}$ away from the centerline.

When instead of focusing into the average velocity values the standard deviation (represented with bars in Fig. 15) of velocity between the selected snapshot datasets is compared, other interesting findings emerge.

In the case of the transverse velocity field the deviation remained quite high along the entire profile except for a reduction near the center, since as previously explained per-

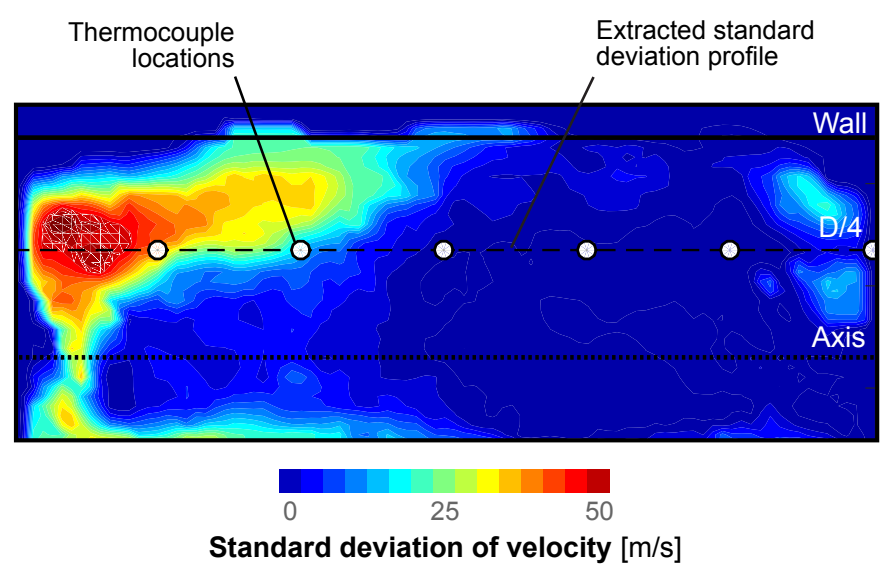

Figure 16: Standard deviation of velocity between selected snapshots in the longitudinal plane, showing the location of the thermocouples used in the temperature measurements. 


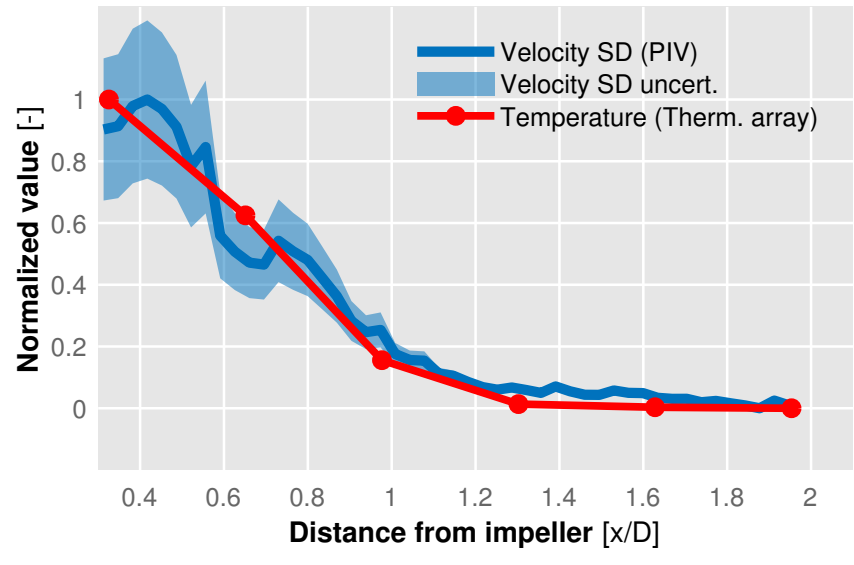

Figure 17: Normalized profile comparison between temperature measurements and velocity standard deviation in the longitudinal plane as seen in Fig. 16.

turbations from the blades in the background and seeding particles lumping together have a higher impact on each individual snapshot. Averaging a large number of them correctly estimates the average velocity but the deviation remains high.

On the other hand, the evolution of the velocity deviation in the case of the longitudinal profile shows a clear difference between a very low deviation upstream and a significant increase in deviation downstream and towards the impeller, which seems well correlated with the increase in temperature caused by the backflow.

In Fig. 16 the spatial distribution of standard deviation has been plotted, showing how its increase is related to the backflow-affected zone. The location of the linear array thermocouples has been added, along with the path of the standard deviation profile selected.

The same axial temperature profile is depicted in Fig. 17 along the normalized magnitude of the velocity standard deviation. Uncertainty of the standard deviation [44] was approximated applying Eq. 5, which for the selected number of samples has less than $4.5 \%$ of error [45], and added to the plot, showing that the temperature profile mostly fits within the uncertainty region.

$$
U_{\bar{\sigma}}=\frac{\sigma}{\sqrt{2(N-1)}}
$$

A very good match between the two distributions is obtained this way, further attesting how the high temperature, reversed flow boosts the variability and complexity of the velocity field near the compressor impeller at unstable working conditions.

This is in line with results of Liu et al. [20] that showed how the standard deviation of temperature, pressure and mass flow measured at selected locations abruptly increased near deep surge conditions, thereby being a valuable indicator to analyze the partially stalled flow instabilities.

\section{Summary}

In this paper an experimental effort with the objective of characterizing the reversed flow of a turbocharger compressor in unstable conditions when this backflow is confined and fully developed in a straight duct has been described.

Prior to the PIV measurements a linear array of thermocouples was installed into the inlet in order to characterize the temperature distribution along the pipe at different operating conditions of shaft speed and air mass flow.

A simple analysis procedure was used and described to determine, using these temperature distributions, the approximate extent that the hot backflow had travelled upstream of the impeller, interpolating in this way a map of the compressor showing the length of the backflow at each condition.

The straight duct containing the thermocouples was then substituted with a transparent glass pipe in order to allow the illumination of the seeded flow with the laser sheet and the imaging of the particles with the camera.

First, an attempt was made to measure the axial velocity field by imaging a axial plane introducing the laser sheet aligned with the turbocharger axis and positioning the camera in a perpendicular way so that images would be captured through the glass duct.

This proved difficult as the oil particles deposited into the walls of the duct, obstructing the view of the camera and enhancing the detrimental reflections that made the correlation difficult or even impossible in some areas. The effect was found to be even worse when conditions close to surge were reached, as the highly rotational backflow forced the oil particles into the walls and also created an stagnation zone where particles formed a liquid film ring.

As a result of these issues, few valid snapshots of the longitudinal plane could be captured, leading to the conclusion that further research of other seeding mechanisms (such as the alumina dispersion used by Wernet [24] or the fluorescent particles used by Wu et al. [27]) or other coating of the pipe (such as the fluorescent coating used by Gancedo et al. [37]) is required if a larger number of snapshots is required.

In any case, the increase of core speed resulting of the reversed flow along the periphery predicted numerically in related investigations $[15,43]$ was observed, and shown to be coherent with the apparent reduction in useful diameter and also with prior temperature measurements.

Comparison with higher mass flow rate conditions allows the authors to conclude that the difference between an stable axial flow field and the unstable one caused by reversed flow in a confined pipe can be characterized through PIV, albeit with difficulty.

A similar comparison between unstable and stable working conditions was performed in the transversal plane, keeping the distance to the impeller equal in both cases. Again a clear difference was observed with a highly rotational flow appearing at unstable conditions.

To test that measurements in that plane can be used as well to estimate the length that the backflow reaches when confined in a straight pipe, an experiment was performed 
measuring three "slices" of the flow field: two inside the predicted length of the backflow and one outside.

This experiment successfully showed a clear difference between the tangential flow field directly caused by the highly rotating backflow and the relatively unaffected field upstream, allowing the conclusion that this slicing method can be successfully used to externally characterize the length and radial distribution of a confined backflow without perturbation as long as images can be captured from the inlet.

Regarding difficulty, measurements in this transversal planes were easier as the images could be captured directly through the inlet pipe mouth instead of through the glass, thereby avoiding the oil fouling problem. However, a higher ensemble of snapshot pairs was needed since the seeding particles tended to lump together and leave gaps in each snapshot.

Finally, a comparison between the PIV results and the previous temperature measurements was performed in order to ensure that both methods were in agreement. Measurements were normalized in order to meaningfully compare the profiles.

Comparison of the radial profile was done extracting data from the slice closer to the compressor, and the closer thermocouple was introduced step by step into the flow until the inlet duct axis. A good agreement between the two results was found, reinforcing the conclusion that the high tangential speed profile measured by PIV is caused by the hot reversed flow and it is accurately capturing its velocity distribution.

Comparison in terms of axial velocity profile was also performed. While the non-disturbed upstream flow and the maximum length of the backflow appeared to match, profiles inside the backflow were more different than in the transversal case, probably due to the aforementioned difficulties regarding the PIV measurement through the glass in the presence confined backflow.

However if the standard deviation of the recorded snapshots is analyzed, a good agreement can be found between the raising in deviation and the raising in temperature, taking advantage of the highly three-dimensional features of the backflow that contrast with the relatively unperturbed characteristics of the upstream flow.

\section{Conclusions}

In conclusion, it has been shown that PIV measurements can be used to successfully characterize the flow field in the presence of a confined backflow in the compressor inlet, with the added advantage of avoiding the disturbances that thermocouples or other sensors can introduce.

Measurements from both axial and transverse planes have allowed to obtain a characterization of the morphology of the reversed flow at different operating conditions. At the selected $140 \mathrm{krpm}$ conditions, reversed flow extended for $\sim 1.3 \mathrm{D}$ an caused a $\sim 30 \%$ reduction of usable radius, more than doubling the centerline velocity as a consequence.
Inside the backflow, high tangential velocity of up to 80 $\mathrm{m} / \mathrm{s}$ was recorded along the periphery, with its intensity diminishing further from the impeller, to the point of vanishing just D/2 upstream of the backflow limit. Diffusion of tangential momentum from the peripheral reversed flow to the core incoming air was shown to be smooth rather than abrupt, imparting rotation to the core up to just $\pm 1 / 8 \mathrm{D}$ from the inlet axis.

PIV results are in good agreement with existing numerical predictions such as those shown in Fig. 1 and with the proxy estimations of the backflow length via temperature profiles, such as those illustrated by Fig. 7. The analysis of velocity deviation demonstrated good potential for detecting the backflow even at the most adverse seeding circumstances.

These findings indicate that future works using PIV would be useful in order to visualize the effect of different inlet configurations designed to mitigate unstable flow issues such as guiding vanes, tapered ducts, nozzles, etc. and to provide better calibration and validation of numerical simulations or other backflow characterization techniques such as the analysis of the inlet temperature field.

\section{Acknowledgements}

This work has been partially supported by Jaguar Land Rover Limited, Abbey Road, Whitley, Coventry CV3 4LF, UK.

The equipment used in this work has been partially supported by the Spanish Ministerio de Economía y Competitividad through grant $n^{\circ}$ DPI2015-70464-R and by FEDER project funds "Dotación de infraestructuras científico técnicas para el Centro Integral de Mejora Energética y Medioambiental de Sistemas de Transporte (CiMeT), (FEDER-ICTS-2012-06)" framed in the operational program of unique scientific and technical infrastructure of the Spanish Ministerio de Economía y Competitividad.

J. García-Tíscar was partially supported through contract FPI-S2-2015-1530 of the Programa de Apoyo para la Investigación y Desarrollo (PAID) of Universitat Politècnica de València.

The authors also wish to thank Mr. Yogesh Narvekar for his valuable contributions, Mr. Carles Oltra for his inestimable assistance during the experimental campaign and Dr. Navarro for his helpful assistance with the illustrative numerical data.

\section{References}

[1] W. Knecht, Diesel engine development in view of reduced emission standards, Energy 33 (2) (2008) 264-271. doi:10.1016/j . energy . 2007. 10.003.

[2] J. W. G. Turner, R. J. Pearson, S. A. Kenchington, Concepts for improved fuel economy from gasoline engines, International Journal of Engine Research 6 (2) (2005) 137-157. doi: 10.1243/146808705X7419.

[3] F. Schumann, F. Sarikoc, S. Buri, H. Kubach, U. Spicher, Potential of spray-guided gasoline direct injection for reduction of fuel consumption and simultaneous compliance with stricter emissions regulations, International Journal of Engine Research 14 (1) (2013) 80-91. doi: $10.1177 / 1468087412451695$. 
[4] Y. Kim, A. Engeda, R. Aungier, G. Direnzi, The influence of inlet flow distortion on the performance of a centrifugal compressor and the development of an improved inlet using numerical simulations, Proceedings of the Institution of Mechanical Engineers, Part A: Journal of Power and Energy 215 (3) (2001) 323-338. doi : 10. 1243/0957650011538550.

[5] A. Stodola, L. Loewenstein, Steam and gas turbines: with a supplement on the prospects of the thermal prime mover, Steam and Gas Turbines: With a Supplement on The Prospects of the Thermal Prime Mover, McGraw-Hill, 1927.

URL https://books.google.es/books? id=CMuEAAAAIAAJ

[6] R. O. Bullock, W. W. Wilcox, J. J. Moses, Experimental and theoretical studies of surging in continuous-flow compressors (NACA Technical Report 861).

URL http: //ntrs.nasa.gov/search. jsp?R=19930091933

[7] D. Fink, N. Cumpsty, E. Greitzer, Surge dynamics in a free-spool centrifugal compressor system, Journal of Turbomachinery 114 (2) (1992) 321-332. doi:10.1115/1.2929146.

[8] D. Evans, A. Ward, Minimizing Turbocharger Whoosh Noise for Diesel Powertrains, SAE Technical Paper 2005-01-2485. doi:10.4271/ 2005- 01 - 2485.

[9] C. Teng, S. Homco, Investigation of Compressor Whoosh Noise in Automotive Turbochargers, SAE Int. J. of Passeng. Cars-Mech. Syst. 2 (1) (2009) 1345-1351. doi: 10.4271/2009-01-2053.

[10] L. Wang, C. Yang, B. Zhao, D. Lao, C. Ma, D. Li, The change of the inlet geometry of a centrifugal compressor stage and its influence on the compressor performance, Journal of Thermal Science 22 (3) (2013) 197-208. doi : 10.1007/s11630-013-0613- 2.

[11] J. Galindo, F. Arnau, A. Tiseira, R. Lang, H. Lahjaily, T. Gimenes, Measurement and modeling of compressor surge on engine test bench for different intake line configurations, SAE Technical Paper (2011-010370). doi: 10.4271/2011-01-0370.

[12] A. Karim, K. Miazgowicz, B. Lizotte, A. Zouani, Computational aeroacoustics simulation of compressor whoosh noise in automotive turbochargers, SAE Technical Paper (2013-01-1880). doi:10.4271/ 2013-01- 1880.

[13] J. Galindo, J. R. Serrano, X. Margot, A. Tiseira, N. Schorn, H. Kindl, Potential of flow pre-whirl at the compressor inlet of automotive engine turbochargers to enlarge surge margin and overcome packaging limitations, International journal of heat and fluid flow 28 (3) (2007) 374-387. doi:10.1016/j.ijheatfluidflow.2006.06.002.

[14] R. P. Tanna, J. Yin, B. Sirakov, V. Barbarie, Development of ported shroud compressor housing with reduced compressor blade pass source acoustic level, in: $9^{\text {th }}$ International Conference on Turbochargers and Turbocharging, 2010.

[15] A. Broatch, J. Galindo, R. Navarro, J. García-Tíscar, A. Daglish, R. Sharma, Simulations and measurements of automotive turbocharger compressor whoosh noise, Engineering Applications of Computational Fluid Mechanics 9 (1) (2015) 12-20. doi : 10. 1080/19942060.2015 1004788.

[16] A. Broatch, J. Galindo, R. Navarro, J. García-Tíscar, Numerical and experimental analysis of automotive turbocharger compressor aeroacoustics at different operating conditions 61 (2016) 245-255. doi: 10. 1016/j. ijheatfluidflow. 2016.04.003.

[17] A. Broatch, J. Galindo, R. Navarro, J. García-Tíscar, Methodology for experimental validation of a cfd model for predicting noise generation in centrifugal compressors, International Journal of Heat and Fluid Flow 50 (2014) 134 - 144. doi: 10.1016/j . ijheatfluidflow. 2014 06.006 .

[18] J. Andersen, F. Lindström, F. Westin, Surge definitions for radial compressors in automotive turbochargers, SAE International Journal of Engines 1 (1) (2009) 218-231. doi : 10.4271/2008-01-0296.

[19] N. Figurella, R. Dehner, A. Selamet, K. Tallio, K. Miazgowicz, R. Wade, Noise at the mid to high flow range of a turbocharger compressor, in: INTER-NOISE and NOISE-CON Congress and Conference Proceedings, Vol. 2012, Institute of Noise Control Engineering, 2012, pp. 8127-8138.

[20] A. Liu, X. Zheng, Methods of surge point judgment for compressor experiments, Experimental Thermal and Fluid Science 51 (2013) 204 213. doi : 10.1016/j . expthermflusci.2013.07.015.

[21] N. Pedersen, P. S. Larsen, C. B. Jacobsen, Flow in a centrifugal pump impeller at design and off-design conditions-part i: particle image velocimetry (piv) and laser doppler velocimetry (ldv) measurements,
Journal of Fluids Engineering 125 (1) (2003) 61-72. doi: 10.1115/1. 1524585.

[22] J. M. F. Oro, E. B. Marigorta, K. M. A. Díaz, R. Ballesteros-Tajadura, Forced and unforced unsteadiness in an axial turbomachine, Experimental Thermal and Fluid Science 33 (3) (2009) 449-459. doi: 10.1016/j . expthermflusci.2008.10.012.

[23] A. K. Vester, S. S. Sattarzadeh, R. Örlü, Combined hot-wire and piv measurements of a swirling turbulent flow at the exit of a $90^{\circ}$ pipe bend, Journal of Visualization (2015) 1-13doi:10.1007/ s12650-015-0310- 1 .

[24] M. Wernet, Development of digital particle imaging velocimetry for use in turbomachinery, Experiments in Fluids 28 (2) (2000) 97-115. doi : $10.1007 / \mathrm{s} 003480050015$.

[25] B. Liu, X. Yu, H. Liu, H. Jiang, H. Yuan, Y. Xu, Application of spiv in turbomachinery, Experiments in fluids 40 (4) (2006) 621-642. doi : 10. 1007/s00348-005-0102- 9.

[26] A. Dazin, G. Cavazzini, G. Pavesi, P. Dupont, S. Coudert, G. Ardizzon, G. Caignaert, G. Bois, High-speed stereoscopic piv study of rotating instabilities in a radial vaneless diffuser, Experiments in fluids 51 (1) (2011) 83-93. doi : 10. 1007/s00348-010-1030-x.

[27] Y. Wu, S. Liu, H. Yuan, J. Shao, Piv measurement on internal instantaneous flows of a centrifugal pump, Science China Technological Sciences 54 (2) (2011) 270-276. doi : 10. 1007/s11431-010-4262-3.

[28] M. Fike, G. Bombek, M. Hriberšek, A. Hribernik, Visualisation of rotating stall in an axial flow fan, Experimental Thermal and Fluid Science 53 (2014) 269-276. doi : 10.1016/j . expthermflusci .2013 . 12.020.

[29] M. P. Wernet, Application of dpiv to study both steady state and transient turbomachinery flows, Optics \& Laser Technology 32 (7) (2000) 497525. doi : 10. 1016/S0030-3992 (00)00090-6.

[30] M. P. Wernet, M. M. Bright, G. J. Skoch, An investigation of surge in a high-speed centrifugal compressor using digital piv, Journal of turbomachinery 123 (2) (2001) 418-428. doi : 10.1115/1. 1343465.

[31] M. Voges, M. Beversdorff, C. Willert, H. Krain, Application of particle image velocimetry to a transonic centrifugal compressor, Experiments in Fluids 43 (2-3) (2007) 371-384. doi : 10. 1007/s00348- 007-0279 - 1.

[32] S. Ohuchida, H. Tamaki, K. Tomoki, K. Yamada, M. Maruyama, Internal flow measurements of turbomachinery using piv, IHI Engineering Review 46 (1) (2013) 22-28.

URL http://www.ihi.co.jp/var/ezwebin_site/storage/ original/application/df5aeb4f6735203337c03f03f7a2f49b.pdf

[33] B. Cukurel, P. B. Lawless, S. Fleeter, Particle image velocity investigation of a high speed centrifugal compressor diffuser: spanwise and loading variations, Journal of Turbomachinery 132 (2) (2010) 021010. doi: 10.1115/1.3104616.

[34] E. Guillou, R. DiMicco, E. Gutmark, A. Mohamed, M. Gancedo, Characterization of a Ported Shroud Compressor using PIV Measurements, Tech. Rep. 2010-01-1225, SAE Technical Paper (2010). doi : 10.4271/ 2010-01- 1225.

[35] E. Guillou, M. Gancedo, R. DiMicco, E. Gutmark, F. Hellstrom, L. Fuchs, A. Mohamed, Surge characteristics in a ported shroud compressor using PIV measurements and large eddy simulation, in: $9^{\text {th }}$ International Conference on Turbochargers and Turbocharging, 2010. doi : 10.1243/ 17547164 C0012010012.

[36] E. Guillou, M. Gancedo, E. Gutmark, A. Mohamed, PIV investigation of the flow induced by a passive surge control method in a radial compressor, Experiments in fluids 53 (3) (2012) 619-635. doi: 10 . 1007/s00348-012- 1310-8.

[37] M. Gancedo, E. Gutmark, E. Guillou, Piv measurements of the flow at the inlet of a turbocharger centrifugal compressor with recirculation casing treatment near the inducer, Experiments in Fluids 57 (2) (2016) 1-19. doi : 10.1007/s00348-015-2105- 5.

[38] F. Hellström, E. Guillou, M. Gancedo, R. DiMicco, A. Mohamed, E. Gutmark, L. Fuchs, Stall Development in a Ported Shroud Compressor using PIV Measurements and Large Eddy Simulation, Tech. rep., SAE Technical Paper 2010-01-0184 (2010). doi : 10.4271/2010-01-0184.

[39] J. Galindo, A. Tiseira, F. J. Arnau, R. Lang, On-engine measurement of turbocharger surge limit, Experimental Techniques 37 (1) (2013) 47-54. doi:10.1111/j.1747-1567.2010.00697.x.

[40] J. Galindo, J. R. Serrano, C. Guardiola, C. Cervelló, Surge limit definition in a specific test bench for the characterization of automotive turbochargers, Experimental Thermal and Fluid Science 30 (5) (2006) 
449-462. doi:10.1016/j.expthermflusci.2005.06.002.

[41] A. Torregrosa, A. Broatch, X. Margot, J. García-Tíscar, Y. Narvekar, R. Cheung, Local flow measurements in a turbocharger compressor inlet, Experimental Thermal and Fluid Science 88 (2017) 542-553. doi:10.1016/j.expthermflusci.2017.07.007.

[42] A. Broatch, S. Ruiz, J. García-Tíscar, F. Roig, On the influence of inlet elbow radius on recirculating backflow, whoosh noise and efficiency in turbocharger compressors, Experimental Thermal and Fluid Science 96 (2018) 224-233. doi:10.1016/j .expthermflusci.2018.03.011.
[43] R. Lang, Contribución a la Mejora del Margen de Bombeo en Compresores Centrífugos de Sobrealimentación, Ph.D. thesis, Universitat Politècnica de València (2011).

URL http : //hdl . handle. net/10251/12331

[44] A. Sciacchitano, B. Wieneke, Piv uncertainty propagation, Measurement Science and Technology 27 (8) (2016) 084006. doi : 10.1088/ $0957-0233 / 27 / 8 / 084006$.

[45] S. Ahn, J. A. Fessler, Standard errors of mean, variance, and standard deviation estimators, Tech. rep., EECS Department, The University of Michigan (2003).

URL https://web.eecs.umich.edu/ fessler/papers/files/tr/ stderr.pdf 